\title{
Outcome in decompensated alcoholic cirrhotic patients with acute variceal bleeding
}

\author{
J E J Krige, U K Kotze, R Sayed, S Burmeister, M Bernon, G Chinnery
}

Background. Variceal bleeding (VB) is the leading cause of death in cirrhotic patients with oesophageal varices. We evaluated the efficacy of emergency endoscopic intervention in controlling acute variceal bleeding and preventing rebleeding and death during the index hospital admission in a large cohort of consecutively treated alcoholic cirrhotic patients after a first variceal bleed.

Methods. From January 1984 to August 2011, 448 alcoholic cirrhotic patients (349 men, 99 women; median age 50 years) with VB underwent endoscopic treatments (556 emergency, 249 elective) during the index hospital admission. Endoscopic control of initial bleeding, variceal rebleeding and survival after the first hospital admission were recorded.

Results. Endoscopic intervention alone controlled VB in 394 patients (87.9\%); 54 also required balloon tamponade. Within 24 hours 15 patients rebled; after 24 hours $61(17 \%, n=76)$ rebled; and $93(20.8 \%)$ died in hospital. No Child-Pugh (C-P) grade A patients died, while 16 grade $\mathrm{B}$ and 77 grade $\mathrm{C}$ patients died. Mortality increased exponentially as the C-P score increased, reaching $80 \%$ when the C-P score exceeded 13.

Conclusion. Despite initial control of variceal haemorrhage, 1 in 6 patients $(17 \%)$ rebled during the first hospital admission. Survival (79.2\%) was influenced by the severity of liver failure, with most deaths occurring in C-P grade $\mathrm{C}$ patients.

S Afr Med J 2012;102(6):554-557.
Variceal bleeding accounts for $70 \%$ of upper gastrointestinal bleeding episodes in patients with portal hypertension. ${ }^{1}$ It significantly influences the natural history of compensated alcgaoholic cirrhosis because a quarter of initial bleeding episodes are fatal, and as many as $70 \%$ of survivors, if inadequately treated, have recurrent variceal bleeding. ${ }^{2}$ Endoscopic control of bleeding is widely used as the emergency treatment of choice for actively bleeding oesophageal varices. $^{3-5}$ Although treatment advances have reduced overall mortality, ${ }^{6,7}$ uncontrolled or recurrent bleeding from varices and the consequences of ensuing liver decompensation remain the most common cause of early death in alcoholic cirrhotic patients. ${ }^{8}$

Despite the general use of endoscopic therapy, there are few robust and validated data on the efficacy of endoscopic control of the index variceal bleed, the frequency of early rebleeding, and survival following the initial bleed in alcoholic cirrhotic patients. ${ }^{6-8}$ Published results are inconsistent and conflicting because of small sample sizes, referral bias, dissimilar study end-points, and differences in patient selection, methods and techniques of endoscopic intervention, and the precise definition of rebleeding., ${ }^{4,9}$ There is consensus that assessment of intervention efficacy in cirrhotic patients with portal hypertension and bleeding oesophageal varices should be based on the control of bleeding and the risks of rebleeding and death as the 3

Department of Surgery, Faculty of Health Sciences, University of Cape Town and Surgical Gastroenterology Unit, Groote Schuur Hospital, Cape Town

J E J Krige, MB ChB, MSc, FACS, FRCS (Ed), FCS (SA)

U K Kotze, RN, RM, CHN, BA CUR

S Burmeister, MB ChB, FCS (SA)

M Bernon, MB ChB, FCS (SA)

G Chinnery, MB ChB, FCS (SA)

School of Public Health and Family Medicine, Faculty of Health Sciences, University of Cape Town

R Sayed, MSc major outcomes. ${ }^{10,11}$ We aimed to evaluate the efficacy of endoscopic intervention in achieving control of acute variceal bleeding and preventing rebleeding and death during the first hospital admission in a large cohort of consecutively treated alcoholic cirrhotic patients with bleeding oesophageal varices.

\section{Methods}

Consecutive adult alcoholic cirrhotic patients with endoscopically proven acute oesophageal variceal bleeding who were admitted to a specialist surgical gastroenterology unit between January 1984 and August 2011 were assessed. These patients received their first emergency and subsequent endoscopic interventions in our unit. Data were recorded prospectively on a standard pro forma and entered into a database maintained by a dedicated research assistant. The diagnosis of cirrhosis was established by liver function tests, ultrasound and portal Doppler assessment, liver biopsy and, in selected patients, hepatic vein wedge pressure measurements. Cirrhosis was considered to be alcohol-related if patients gave a history of sustained heavy alcohol consumption over several years with corroborative liver histological evidence and exclusion of other causes. The study was approved by the departmental and institutional ethics and research committees.

During the 332-month study period, 693 consecutive adult patients were treated for oesophageal variceal bleeding; 245 had non-alcoholic causes of portal hypertension and were excluded; 469 had portal hypertension caused by alcohol-induced cirrhosis. Of these, 21 patients were not included because they also had positive hepatitis $\mathrm{B}$ or $\mathrm{C}$ viral markers. This study is based on data in the remaining 448 patients with alcoholic cirrhosis and proven oesophageal variceal bleeding who received endoscopic intervention for bleeding.

Control of variceal bleeding was evaluated at 2 time points: initial control during the first presentation and rebleeding during the index hospital admission. The primary clinical endpoints were: $(i)$ failure to control variceal bleeding during the initial endoscopic intervention, (ii) rebleeding after initial endoscopic control, and (iii) mortality during the initial hospital admission.

Injection sclerotherapy was used as described previously. ${ }^{12,13}$ Both diagnostic endoscopy and injection sclerotherapy were performed 
Table 1. Child-Pugh grade, blood transfusion, balloon tube and vasopressin requirements to control acute variceal bleeding during the index hospital admission

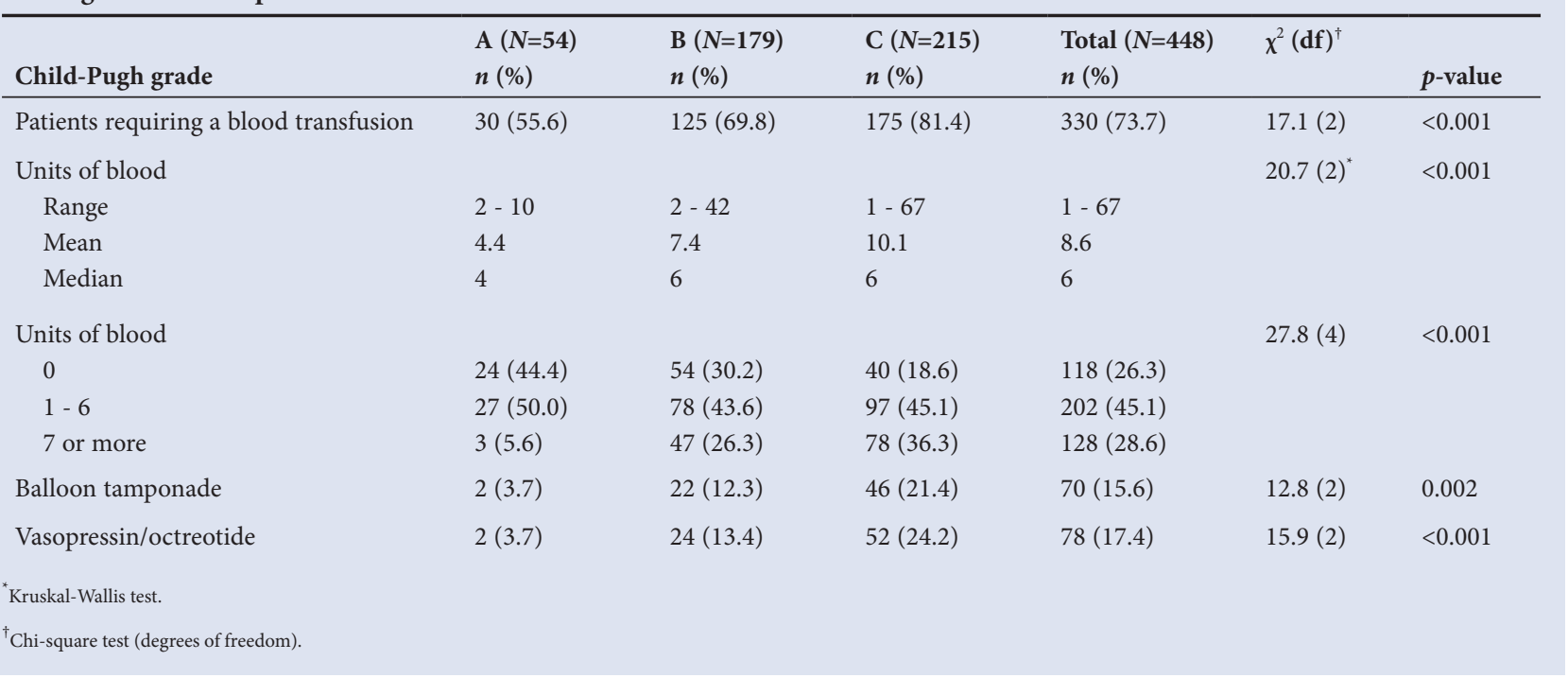

using a fibreoptic endoscope (GIF 1T20 or K10, Olympus Corp) during the first decade of the study and video-endoscopy during the last decade. The sclerosant, $5 \%$ ethanolamine oleate, was injected using a combined intra- and paravariceal technique. ${ }^{12,13}$

The original single-shot endoscopic variceal banding device (C R Bard, Tewksbury, MA) was used initially when banding was introduced in $1994 . .^{13,14}$ On average, a total of 6 bands were applied during the initial endoscopic banding session, and progressively fewer bands were required during subsequent sessions as the varices decreased in size. The technical disadvantage of the single band applicator is that the endoscope has to be removed and reinserted repeatedly with a new band attached for each individual variceal ligation. The introduction of the newly designed multiband ligator overcame these disadvantages with 6 mounted bands which are individually activated by a drawstring attached to a trigger unit positioned in the biopsy channel port of the endoscope, allowing repeated firing of the bands and obviating the need for an overtube..$^{14,15}$ Equipment used included either an Olympus XQ 240 or 260 endoscope, and a 6-shooter multiband ligator (Wilson-Cook Medical).

Time zero was defined as the time of hospital admission. Failure to control bleeding was defined as continued bleeding despite endoscopic injection and the use of balloon tamponade. Rebleeding was defined as any episode of upper gastrointestinal bleeding that occurred after the initial bleed had been successfully controlled by endoscopic therapy. Rebleeding was treated by urgent endoscopy and endoscopic control of bleeding if it originated in patent residual varices. Patient data were evaluated only during the initial hospital admission. All bleeding and complications related to endoscopic intervention and deaths during this period were recorded. Rebleeding was evaluated in 3 specific categories: (i) uncontrolled bleeding during the index scope with failure of endoscopic treatment, (ii) continued or early rebleeding within 24 hours of the initial endoscopic treatment, requiring a second endoscopy, and (iii) rebleeding more than 24 hours after the index endoscopic treatment.

Data were stored on a spreadsheet registry and analysed using STATA 11.0 (StataCorp). Associations between categorical variables were analysed using Pearson's chi-square test and the chi-square for linear trend for ordinal variables. Prevalence ratios (PRs) and 95\% confidence intervals (CIs) were estimated from binomial regression models. The Kruskal-Wallis test was used to compare units of blood

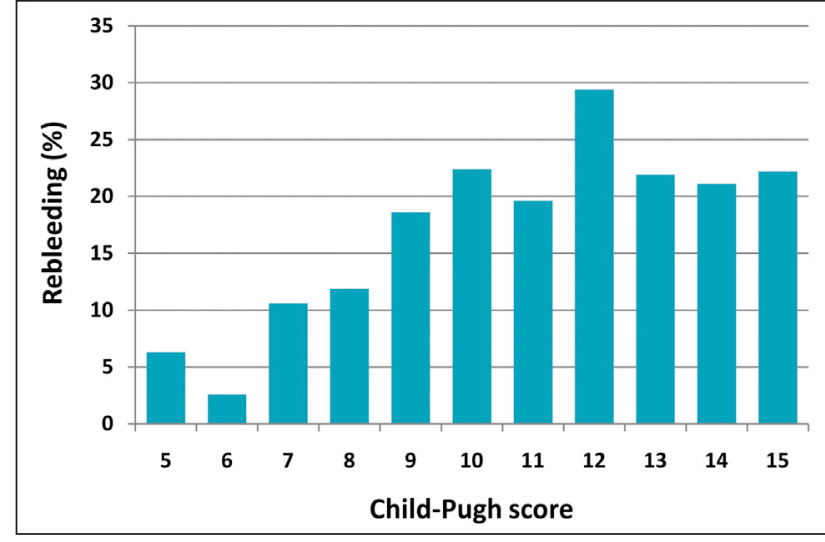

Fig. 1. Rebleeding according to Child-Pugh score.

in each of the 3 Child-Pugh (C-P) grades. For all analyses, a $p$-value $<0.05$ and $95 \%$ CI that did not span unity were considered the thresholds of statistical significance.

\section{Results}

The 448 patients evaluated included 349 men and 99 women of median age 50 years (range 24 - 87); 54 were C-P grade A, 179 were $\mathrm{C}-\mathrm{P}$ grade $\mathrm{B}$ and 215 were $\mathrm{C}-\mathrm{P}$ grade $\mathrm{C}$ when assessed on first hospital admission (Table 1). A blood transfusion was required by 330 patients during the initial admission. Balloon tamponade with a Sengstaken-Blakemore or Minnesota tube was used in 70 patients and a vasopressin or octreotide infusion was used in 78 (Table 1); in 28 , both a balloon tube and vasopressin were used. Compared with grade $\mathrm{B}$ patients, significantly more $\mathrm{C}-\mathrm{P}$ grade $\mathrm{C}$ patients required a major blood transfusion ( 7 or more units), the use of balloon tube and vasopressin or octreotide to control variceal bleeding (Table 1). Median hospital stay for the 355 patients who survived the first admission was 7 days (range 1 - 39).

All 448 patients underwent emergency endoscopy which confirmed oesophageal varices as the source of bleeding. In 440/448 patients, endoscopic intervention was used to control variceal bleeding at the index endoscopy session; 349 had sclerotherapy (median volume $14 \mathrm{ml}$, range $1-40$ ) as the initial endoscopic intervention and 86 had endoscopic band ligation of varices (median 5 bands, range 


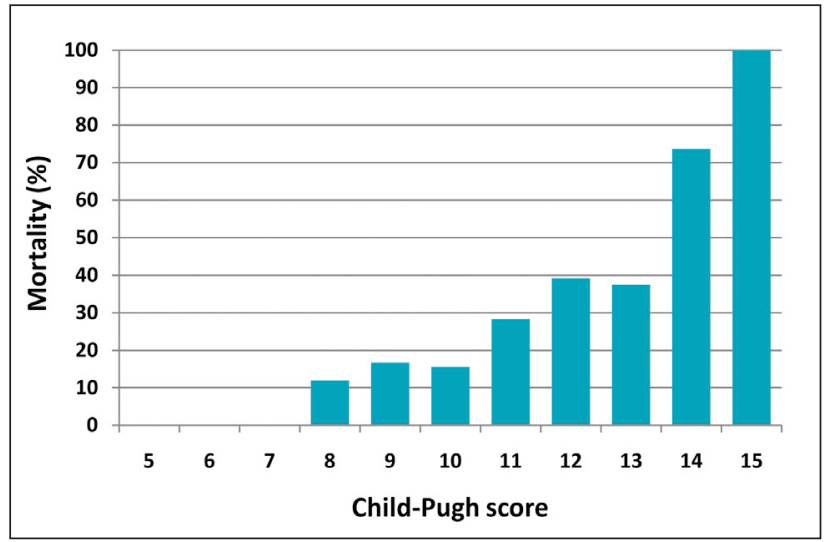

Fig. 2. Mortality according to Child-Pugh score.

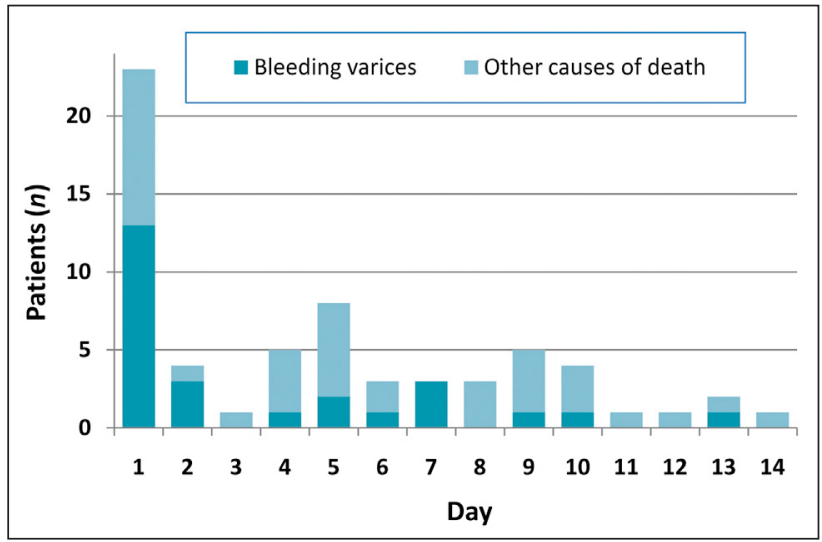

Fig. 3. Hospital deaths (days 1 - 14).

1 - 10). Five patients had both endoscopic banding and injection sclerotherapy during the first endoscopy session in an attempt to control variceal bleeding. In 394 (87.9\%) patients, control of variceal bleeding using endoscopic intervention alone was regarded as adequate haemostasis; in $8(1.8 \%)$ variceal bleeding was profuse and visibility obscured, preventing safe endoscopic intervention and a Sengstaken balloon tube was inserted to control bleeding, followed by repeat endoscopy 12 hours later. In $46(10.3 \%)$ patients who had initial endoscopic intervention, the endoscopist was concerned about the adequacy of the intervention and placed a Sengstaken tube on completion of the procedure. A total of $54(12.1 \%)$ patients thus had a Sengstaken tube placed during treatment of the initial bleed. In $8(1.8 \%)$ patients, variceal bleeding persisted despite pharmacological and endoscopic therapy and balloon tamponade. A total 805 endoscopic treatments were performed during the index hospital admission in the 448 patients, including 556 emergency and 249 elective procedures.

Within 24 hours of the index intervention, 15 (3.3\%) patients underwent urgent repeat endoscopy because of continued $(n=8)$ or early $(n=7)$ rebleeding. A further $61(13.6 \%)$ rebled after 24 hours

Table 2. Rebleeding according to Child-Pugh grade

\begin{tabular}{llllll}
\hline & Total & Rebleeding & & & \\
Class & $(\boldsymbol{N})$ & $\boldsymbol{n}(\%)$ & PR & $\boldsymbol{p}$-value & $\mathbf{9 5 \%}$ CI \\
\hline A & 54 & $2(3.7)$ & - & - & - \\
B & 179 & $24(13.4)$ & 3.6 & 0.074 & $0.88-14.83$ \\
C & 215 & $50(23.3)$ & 6.3 & 0.009 & $1.58-25.0$
\end{tabular}

Table 3. Mortality according to Child-Pugh grade

\begin{tabular}{llllll}
\hline Class & $\begin{array}{l}\text { Total } \\
(\boldsymbol{N})\end{array}$ & $\begin{array}{l}\text { Died } \\
\boldsymbol{n}(\%)\end{array}$ & $\mathbf{P R}$ & $\boldsymbol{p}$-value & $\mathbf{9 5 \%}$ CI \\
\hline A & 54 & $0(0)$ & - & - & - \\
B & 179 & $16(8.9)$ & - & - & - \\
C & 215 & $77(35.8)$ & 4.0 & $<0.001$ & $2.43-6.61$
\end{tabular}

and required further emergency endoscopic intervention to achieve definitive variceal haemostasis (Fig. 1). Six patients required a transjugular intrahepatic portosystemic shunt (TIPS) as a salvage procedure for uncontrolled bleeding during the index admission; 2 died of multi-organ failure. A further 12 required a TIPS because of refractory variceal bleeding on subsequent admission. There was a significant upward trend in the proportion of rebleeding according to C-P grade $(p<0.001)$ (Table 2): $26(11.2 \%)$ of the 233 patients in grades A and B rebled compared with 50 (23.5\%) of the 215 patients in grade $\mathrm{C}(\mathrm{PR}=6.3,95 \% \mathrm{CI} 1.58-25.00, p<0.009)$.

Ninety-three $(20.8 \%)$ patients died during the initial hospital admission (Table 3 ). There was a strong correlation between mortality and C-P score $\left(\mathrm{r}_{\mathrm{s}}=0.97, p<0.001\right): 74 \%$ and $100 \%$ mortality among patients with a C-P score of 14 and 15, respectively (Fig. 2). Median time to death was 7 days (range 0 - 52). There was a total mortality of $64(14.3 \%)$ during the first 2 weeks (Fig. 3), with $23(5.1 \%)$ patients dying within 24 hours of admission and 41 within 5 days. No C-P grade A patients died, 16 grade $B$ patients died, and 77 grade $C$ patients died. Liver failure was the most common cause of death in 40 patients. Eleven patients died of liver and renal failure and 8 died of pneumonia and respiratory failure. Death in 33 patients was a consequence of continued or recurrent variceal bleeding. Survival during the initial hospital admission in C-P grade A patients was $100 \%$, in grade B patients $91.1 \%$, and in grade C patients $64.2 \%$ (Fig. 3). Significantly more grade $C$ patients than grade $B$ patients died $(\mathrm{PR}=4.0,95 \%$ CI $2.4-6.6, p<0.001)$ (Table 3).

Minor sclerotherapy and banding side-effects were common after acute intervention for bleeding, including dysphagia, transient fever, pulmonary atelectasis, pleural effusions and oesophageal mucosal ulceration. Eight $(1.8 \%)$ patients had life-threatening complications after sclerotherapy, 3 developed a deep ulcer with a contained oesophageal perforation at the injection site, and a free oesphageal perforation occurred in 5 patients as a complication of emergency sclerotherapy during active variceal bleeding. Four of the 5 patients with sclerotherapy-induced perforations died, 3 due to recurrent variceal bleeding and 1 as a result of progressive liver and renal failure.

\section{Discussion}

Endoscopy is essential in the modern multidisciplinary management of variceal bleeding. ${ }^{4}$ This study demonstrated that endoscopic therapy was effective in controlling the initial acute variceal bleed and that ultimate survival was influenced by rebleeding and underlying liver reserve. However, despite urgent endoscopic and pharmacological therapy, variceal bleeding is reported to recur in up to $20 \%$ of patients after initial endoscopic intervention. ${ }^{10}$ Early variceal rebleeding also significantly increases the risk of death within 6 weeks of the initial bleed. ${ }^{3,7,10}$ Prevention of rebleeding is a crucial element of management, considering the frequency of rebleeding after initial control. ${ }^{9}$ The lack of a universally implemented definition of failure of secondary prophylaxis has resulted in differences in the published incidence of rebleeding. This has hampered the interpretation of outcomes 
research and hindered accurate comparisons. We demonstrated that, although initial endoscopic intervention controlled acute variceal bleeding in $98.2 \%$ of patients, $17 \%$ rebled during the index hospital admission. The rebleeding rate in our study is lower than the $21 \%$ and $26 \%$ reported by others, ${ }^{16,17}$ despite our strictly observed and prospectively documented definition of rebleeding and the high-risk alcoholic cirrhotic cohort.

Evidence suggests that liver decompensation is a reliable predictor of rebleeding and mortality. Severity of liver disease, quantified as C-P and Meld scores or as individual components, is widely recognised as a robust independent predictor of early failure of bleeding control. ${ }^{3,7,8}$ Active bleeding at initial endoscopy is an important risk factor for early failure in several studies. ${ }^{3,7,8}$ The highest mortality occurs during the first bleed. As in previous studies, ${ }^{5,8}$ our data confirmed that the first 5 days after admission represent the most crucial time period for determining prognosis (41 of 93 deaths occurred during this time). Up to one-third of deaths related to acute variceal bleeding are secondary to uncontrolled or recurrent bleeding, and most of the remaining cases are due to sepsis and liver and renal failure. ${ }^{1,2,4}$ The risk of death after acute variceal bleeding shows an evolution similar to that of rebleeding, peaking during the first 24 hours, and declining after 5 days and returning to the baseline after 6 weeks. ${ }^{16}$

Our observations and those of others show that the efficacy of endoscopic intervention in controlling acute oesophageal variceal bleeding and mortality from bleeding are closely related to the severity of the underlying liver disease. ${ }^{3,5,8}$ As anticipated, mortality in our study increased exponentially as liver reserve diminished. There were no deaths in C-P grade A patients and, in this cohort with preserved liver function, early mortality was not influenced by the severity of the bleeding episode. An earlier study ${ }^{10}$ and the current data demonstrated that C-P grade $\mathrm{C}$ patients, who have the least hepatic reserve, were more likely to have a major bleed and require pitressin or octreotide and balloon tamponade in addition to sclerotherapy to control acute bleeding. Our study shows that in this high-risk group of alcoholic cirrhotic patients with liver decompensation aggravated by a significant variceal bleed, despite successful endoscopic control of the bleeding, progressive liver failure ensued with high mortality rates, especially in patients with C-P scores exceeding 13 , who had a greater than $80 \%$ mortality.

This study had several limitations. The management of variceal bleeding has substantially advanced since the inception of the study, e.g. the use of variceal ligation in favour of sclerotherapy, improved pharmacotherapy with the replacement of vasopressin with octreotide, routine antibiotic prophylaxis, and enhanced critical care. These changes will not have influenced data analysis, as this was a longitudinal cohort study. A major strength was that the study was conducted in a single centre in a defined population of consecutive patients; this eliminates potential bias based on selection of treatment. To provide the highest possible level of uniformity and to minimise differences in the zero-time entry, only patients who received their initial and subsequent treatment in our unit were studied. The study design minimised possible bias that may have arisen from patient selection, referral practices and local variations in treatment strategies. Using rebleeding and death as the main outcomes provided consistent and objective end-points. The study robustness was enhanced by the prospective data collection, complete follow-up and restriction of subjects to those with alcohol-induced cirrhosis. The inception cohort were at a well-defined stage in the course of cirrhosis, providing an homogenous study population and avoiding the error of over-fitting which has influenced previous studies.

\section{Conclusions}

This study has established the outcome of decompensated alcoholic cirrhotic patients after the index variceal bleed. Consistent with previous studies, ${ }^{3,7,8}$ we found that the C-P score was an efficient tool for predicting overall in-hospital mortality. We established that C-P grade A patients respond well to current therapies, with no deaths. However, despite recent improvement in overall survival, mortality remains high in $\mathrm{C}-\mathrm{P}$ grade $\mathrm{C}$ patients, who die from early rebleeding or subsequently from the detrimental systemic consequences that lead to progressive liver function deterioration. While this study demonstrated that endoscopic management is effective in controlling the initial bleed in alcoholic cirrhotic patients, rebleeding occurs in up to $20 \%$ of patients, some of whom may require rescue intervention. These results have important prognostic implications and show that the C-P score does not effectively predict failure to control bleeding. Knowledge of predictive factors to identify those at risk of early rebleeding could allow the effective triage of patients to implement the early use of aggressive salvage therapy. The paucity of accurate and robust data predicting the likelihood of variceal rebleeding warrants further research to define the optimal evidencebased standard of care in this high-risk cohort of patients.

\section{References}

1. D'Amico G, De Franchis R. Upper digestive bleeding in cirrhosis. Post-therapeutic outcome and prognostic indicators. Hepatology 2003;38:599-612. [http://dx.doi.org/10.1053//hep.2003.50385]

. Bornman PC, Krige JE, Terblanche J. Management of oesophageal varices. Lancet 1994;343:1079-1084. Bornman PC, Krige JE, Terblanche J. Management of

Sharara AI, Rockey DC. Gastroesophageal variceal hemorrhage. N Engl J Med 2001;345:669-681. Sharara AI, Rockey DC. Gastroesophagen

[http://dx.doi.org/10.1056/NEJMra003007]

Krige JEJ, Shaw JM, Bornman PC. The evolving role of endoscopic treatment for bleeding esophageal varices. World J Surg 2005;29:966-973. [http://dx.doi.org/10.1007/s00268-005-0138-2 ]

5. Triantos CK, Goulis J, Patch D, et al. An evaluation of emergency sclerotherapy of varices in randomized trials: looking the needle in the eye. Endoscopy 2006;38:797-807. [http://dx.doi org/10.1055/s-2006-944566

6. Garcia-Tsao G, Sanyal AJ, Grace ND, Carey WD. Prevention and management of gastroesophagea varices and variceal hemorrhage in cirrhosis. Am J Gastroenterol 2007;102:2086-2102. [http://dx.doi. org/10.1111/j.1572-0241.2007.01481.x]

Jalan R, Hayes PC. UK guidelines on the management of variceal haemorrhage in cirrhotic patients. Gut 2000;46:Suppl 3-4, III1-III15. [http://dx.doi.org/10.1136/gut.46.suppl_3.iiil]

8. D’Amico G, Garcia-Tsao G, Pagliaro L. Natural history and prognostic indicators of survival in D'Amico G, Garcia-Tsao G, Pagliaro L. Natural history and prognostic indicators of survival cirrhosis: a syste

jhep. 200 .

Krige JEJ, Beningfield SJ, Shaw J. Management of bleeding oesophageal varices. In: Johnson C, Taylor I eds. Recent Advances in Surgery. 30th ed. London: Royal Society of Medicine, 2007:105-125.

10. Krige JE, Shaw JM, Bornman PC, Kotze UK. Early rebleeding and death at 6 weeks in alcoholic cirrhotic patients with acute variceal bleeding treated with emergency endoscopic injection sclerotherapy. S Af J Surg 2009; $47: 76-79$

11. Thomsen BL, Sørensen TI. Analysis of the treatment effect on recurrent bleeding and death in patients with cirrhosis and esophageal varices: multistage competing-risks model compared to conventiona methods. The Copenhagen Esophageal Varices Sclerotherapy Project. J Hepatol 1998;28:107-114.

12. Krige JEJ, Terblanche J. Injection sclerotherapy of oesophageal varices. In: Carter D, Russell RCG, Pit H, Bismuth H, eds. Rob and Smith's Operative Surgery. Surgery of the Liver, Pancreas and Bile Ducts. 5th ed. London: Chapman \& Hall Medical, 1996:163-172.

13. Krige JEJ, Bornman PC. Endoscopic therapy in the management of esophageal varices: injection sclerotherapy and variceal ligation. In: Blumgart L, ed. Surgery of the Liver, Biliary Tract and Pancreas. 4 th ed. Philadelphia: Saunders Elsevier, 2007:1579-1593.

14. Krige JEJ, Bornman PC. Endoscopic therapy in the management of esophageal varices: injection sclerotherapy and variceal ligation. In: Fischer JE, ed. Mastery of Surgery. 5th ed. Philadelphia: Lippincott Williams \& Wilkins, 2006:1367-1378.

15. Tait IS, Krige JE, Terblanche J. Endoscopic band ligation of oesophageal varices. Br J Surg 1999;86:437446. [http://dx.doi.org/10.1046/j.1365-2168.1999.01109.x]

16. Augustin S, Muntaner L, Altamirano JT, et al. Predicting early mortality after acute variceal hemorrhage based on classification and regression tree analysis. Clin Gastroenterol Hepatol 2009;7:1347-1354 [http://dx.doi.org/10.1016/j.cgh.2009.08.011]

17. Hobolth L, Krag A, Bendtsen F. The recent reduction in mortality from bleeding oesophageal varices is primarily observed from Days 1 to 5 . Liver Int 2010;30:455-462. [http://dx.doi.org/10.1111/j.14783231.2009.02169.x]

Accepted 27 January 2012. 